\title{
Caracterización del proceso de fabricación de macroporos sobre sustrato de silicio por método electroquímico
}

\author{
Characterization of fabrication process of \\ micropores on silicon substrate by \\ electrochemical method
}

\author{
Manuel Der ${ }^{1}$, Carol Olmos ${ }^{2}$, Gustavo Rosero ${ }^{2}$, Itzel Santizo ${ }^{2}$, \\ Tamara Fernandez ${ }^{2}$, Maria Dieguez ${ }^{3}$, Francisco $\mathrm{Sacco}^{3}$, \\ Pablo Granell ${ }^{4}$, Federico Golmar ${ }^{4}$, \\ Betiana Lerner ${ }^{2}$, Carlos Lasorsa ${ }^{2}$, \\ Maximiliano Perez ${ }^{2}$
}

\footnotetext{
${ }^{1}$ Departamento de Física, Facultad de Ciencias Exactas y Naturales, Universidad de Buenos Aires, Cuidad Universitaria, Buenos Aires 1428, Buenos Aires, Buenos Aires, Argentina

${ }^{2}$ Universidad Tecnológica Nacional, Facultad Regional Haedo, Grupo de Ingeniería de Recubrimientos especiales y nanotecnología (IREN), Sarmiento, Buenos Aires, Buenos Aires, Argentina.

e-mail: carolmaritza1@gmail.com, gustavorosero@gmail.com, itzelsantizo@gmail.com, betianalerner@gmail.com, lasorsa@cnea.gov.ar, max @fullgen.com.ar

e-mail: manu.der.93@gmail.com

${ }^{3}$ Instituto de Genética "Ewald A. Favret" CICVyA-INTA CASTELAR, Castelar, Buenos Aires, Buenos Aires, Argentina. e-mail: dieguez.maria@inta.gob.ar, sacco.francisco@inta.gob.ar

${ }^{4}$ Instituto Nacional de Tecnología Industrial, Buenos Aires, Argentina.

e-mail: pgranell@inti.gob.ar, fgolmar@inti.gob.ar
}

\section{RESUMEN}

En el presente trabajo se estudió el proceso de fabricación de una serie de macroporos sobre obleas de silicio cristalino mediante la técnica wet etching. Se evaluó la incidencia de distintos factores como el voltaje, la temperatura y el agente de frenado sobre las características específicas de la formación. A partir de los datos obtenidos de la evolución de las corrientes fue posible estandarizar el proceso y determinar el momento de formación del poro, esencial en cuanto a la disponibilidad de un método compatible con las exigencias de la industria. Finalmente, se concluyó que para la fabricación de poros en forma controlada, las condiciones óptimas corresponden a una temperatura de $84{ }^{\circ} \mathrm{C}, \mathrm{HCl}$ como agente de frenado y voltajes de $0,1 \mathrm{~V}, 0,5 \mathrm{~V}$ y $1 \mathrm{~V}$ respectivamente. Los anteriores resultados son de gran importancia en los campos de la medicina y la biología en relación a la utilidad de los poros como dispositivos de sensado.

Palabras clave: macroporos, sensores biológicos, wet etching, silicio.

\begin{abstract}
In the present work, the fabrication process of a set of micropores on crystalline silicon wafers manufactured through wet etching technique was studied. The influence of different control factors such as voltage, temperature and braking agent on the specific characteristics of formation was evaluated. An exhaustive analysis of the evolution of electric currents during the fabrication made possible to standardize the process and determine the pore-formation time, essential feature considering the exigent requirements of industry. Finally, it was concluded that optimum conditions for a controlled fabrication of pores correspond to a temperature of $84{ }^{\circ} \mathrm{C}$, $\mathrm{HCl}$ as a braking agent and voltages of $0,1 \mathrm{~V}, 0,5 \mathrm{~V}$ and $1 \mathrm{~V}$ respectively. The above results are of great importance in different fields, such as biology or medicine, in relation to the utility of pores as sensing devices.
\end{abstract}

Keywords : macropores, biological sensors, wet etching, silicon. 


\section{INTRODUCTION}

Durante la última década las investigaciones en las áreas de la nanotecnología y la nanobiotecnología han motivado al desarrollo de dispositivos con tamaños en el orden de los nanómetros. Actualmente, uno de los grandes intereses se centra en la fabricación de macroporos y nanoporos que permitan el sensado de células y moléculas como nucleotidos, enantiomeros, polimeros como PEG (polietilengrlicol), RNA, DNA y polipéptidos. Esta línea de investigación representa perspectivas positivas en cuanto a la secuenciación de ADN de tercera generación y el diagnóstico médico [1].

Entre los métodos que reporta la literatura para la fabricación de poros se encuentran metodologías que hacen uso de la microscopía electrónica [2-6], métodos de etching químico [7,8] y métodos electroquímicos [9-13]. La fabricación de nanoporos mediante técnicas de microscopía electrónica resulta ser muy útil y permite tener un gran control en relación al tamaño y localización del poro. No obstante, estas tecnologías son costosas y producen poros de alta fragilidad que presentan complicaciones al momento de adaptarlos a las exigencias masivas de la industria médica y biológica, así como a los sucesivos traslados y manipulaciones de los que deberán ser objeto. En cuanto al método electroquímico, este consiste en la aplicación de un voltaje durante el proceso de etching químico controlado, seguido de una neutralización mediada por un agente de frenado. Entre las ventajas de los poros obtenidos mediante la técnica se destacan: alta resistencia, geometría controlada, estabilidad, y control en los tamaños [9-13]. Además, la fabricación puede llevarse a cabo en un laboratorio sin instalaciones costosas.

Para la fabricación de poros mediante el método electroquímico se usan generalmente sustratos de silicio cristalino. Dicho material se expone localmente a un agente como ácido fluorhídrico, hidróxido de sodio, hidróxido de potasio, tetrametil amonio hidróxido, entre otros, que lo atacan químicamente. El etching químico con $\mathrm{KOH}$ ha sido especialmente estudiado debido a su bajo costo, alta relación de ataque (100)/(111) , su naturaleza no tóxica y facilidad de manipulación en laboratorio [7,14]. Durante el proceso, átomos de silicio son arrancados de la estructura cristalina al entrar en contacto con los hidróxidos presentes en el medio. De esta manera, cuando se expone al ataque químico una región acotada sobre la superficie del material, se genera un avance de características anisótropas en relación a la reacción sobre cada plano cristalino. En particular, para el plano $<111>$ se registran velocidades menores en comparación con el plano < $100>$ [15]. De este modo, el ataque químico del sustrato resulta en una pirámide de base cuadrada de unos $54,74^{\circ}$ de inclinación [16].

Sobre la base de las características particulares del método electroquímico, este artículo presentará la caracterización de la fabricación de macroporos bajo diferentes condiciones de control. Se indicarán los resultados del proceso teniendo en cuenta la incidencia de los siguientes parámetros en cuestiones específicas de la formación; a) potencial $(0,1,0,5$ y $1 \mathrm{~V})$, b), agente de frenado (ácido clorhídrico, ácido fórmico) y c) temperatura $\left(65^{\circ} \mathrm{C}\right.$ y $\left.84^{\circ} \mathrm{C}\right)$. A partir del estudio de cada uno de los parámetros mencionados es posible obtener las condiciones óptimas del proceso y al mismo tiempo extraprolar el procedimiento para la fabricación de nanoporos. Cabe resaltar que hasta el momento no se ha reportado la caracterización del proceso de formación de poros bajo las condiciones establecidas en este trabajo.

\section{MATERIALES Y MÉTODOS}

Para la fabricación de los poros se utilizaron obleas de silicio cristalino de $520 \mu \mathrm{m}$ de espesor (Virginia Semiconductor In.) orientadas según el plano $<100>$ de la estructura cristalográfica. Las obleas de silicio fueron recubiertas por una capa de $200 \mathrm{~nm}$ de $\mathrm{Si}_{3} \mathrm{~N}_{4}$ mediante el método de Plasma Enhanced Chemical Vapor Deposition (PECVD) a $600{ }^{\circ} \mathrm{C}$. Como primera etapa del proceso, se llevó a cabo la remoción localizada de nitruro de silicio $\left(\mathrm{Si}_{3} \mathrm{~N}_{4}\right)$ por medio de ablación láser de ventanas cuadradas convenientemente localizadas sobre la superficie de la oblea. El recubrimiento de $\mathrm{Si}_{3} \mathrm{~N}_{4}$, resistente al wet etching, posibilita la localización precisa de la zona de ataque, y en consecuencia el control de la posición del poro resultante (Figura 1) [16].

En una segunda etapa se lleva a cabo un proceso de fast wet etching con una solución $\mathrm{KOH}$ (aq) $7 \mathrm{M}$ a $80^{\circ} \mathrm{C}$ sobre ambos lados de la oblea, que finaliza cuando la separación mínima entre los hemisferios se encuentra en el orden de 100 y $200 \mu \mathrm{m}$ (figura 1.4). 


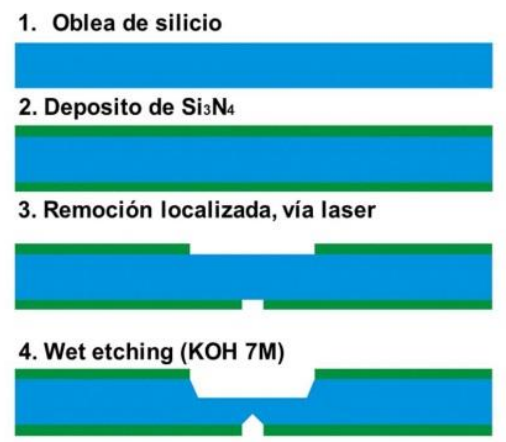

Figura 1: Ilustración esquemática de las etapas iniciales del proceso de formación de poros. 1.1) Oblea de silicio, 1.2) Oblea de silicio con deposición de nitruro de silicio, 1.3) Remoción localizada de nitruro de silicio y 1.4) Wet etching con KOH 7M.

Posteriormente, se realiza un segundo ataque químico en presencia de potencial y temperatura, que culmina con la apertura del poro y el mecanismo de frenado autocontrolado por la neutralización de los reactivos. Para ello, como se puede ver en la Figura 2 se utiliza una celda en la que se ubica en un lado una solución de $\mathrm{KOH} 4 \mathrm{M}$ y en el otro lado el agente de frenado ( $\mathrm{HCl} 12$ M ó HCOOH 30 M según corresponda). En cada compartimiento se introduce un electrodo de platino el cual se conecta a con un dispositivo Keithley $2612 b$ Single and dual channel scalable sourcing and measurement (Keithley Instruments), que entrega el voltaje indicado y es capaz de discriminar corrientes del orden de los 0,1 pA y tensiones de hasta $0,1 \mu \mathrm{V}$. El mismo se controla por medio de conexión USB con el software Test Script Builder (TSB), y se configura para tomar mediciones de corriente en el tiempo con una frecuencia de $0,1 \mathrm{~Hz}$. En esta etapa el encuentro de ambas pirámides invertidas da lugar a la formación del poro y al frenado químico, que disminuye la velocidad del wet etching. Cabe resaltar que previamente nuestro grupo había reportado el proceso de formación de nanoporos bajo condiciones de $\mathrm{KOH} 4 \mathrm{M}$, sin aplicar voltaje al procedimiento [7]. Finalmente, para concluir con el proceso, la oblea es sometida a un lavado con solución piraña (mezcla de $\mathrm{H}_{2} \mathrm{SO}_{4} \mathrm{y} \mathrm{H}_{2} \mathrm{O}_{2}$ en proporción 3:1) para remover las sales remanentes que quedan adheridas a la superficie. Las obleas se codificaron de acuerdo al agente de frenado utilizado, la temperatura y las condiciones de voltaje aplicadas en el proceso respectivamente. La nomenclatura corresponde con la codificación presentada en la tabla 1.

Tabla 1: Codificación de las obleas y condiciones de fabricación de poros

\begin{tabular}{l|l|l|l}
\hline CODIFICACIÓN & TEMPERATURA $\left({ }^{\circ} \mathbf{C}\right)$ & AGENTE DE FRENADO & VOLTAJE APLICADO $(\mathbf{V})$ \\
\hline C-84-1 & $84 \pm 1$ & $\mathrm{HCl}$ & 1 \\
\hline C-84-0.5 & $84 \pm 1$ & $\mathrm{HCl}$ & 0,5 \\
\hline C-84-0.1 & $84 \pm 1$ & $\mathrm{HCl}$ & 0,1 \\
\hline F-84-1 & $84 \pm 1$ & $\mathrm{HCOOH}$ & 1 \\
\hline F-84-0.5 & $84 \pm 1$ & $\mathrm{HCOOH}$ & 0,5 \\
\hline F-84-0.1 & $84 \pm 1$ & $\mathrm{HCOOH}$ & 0,1 \\
\hline C-65-1 & $65 \pm 1$ & $\mathrm{HCl}$ & 1 \\
\hline C-65-0.5 & $65 \pm 1$ & $\mathrm{HCl}$ & 0,5 \\
\hline C-65-0.1 & $65 \pm 1$ & $\mathrm{HCl}$ & 0,1 \\
\hline F-65-1 & $65 \pm 1$ & $\mathrm{HCOOH}$ & 1 \\
\hline F-65-0.5 & $65 \pm 1$ & $\mathrm{HCOOH}$ & 0,5 \\
\hline F-65-0.1 & $65 \pm 1$ & $\mathrm{HCOOH}$ & 0,1 \\
\hline
\end{tabular}


(b)

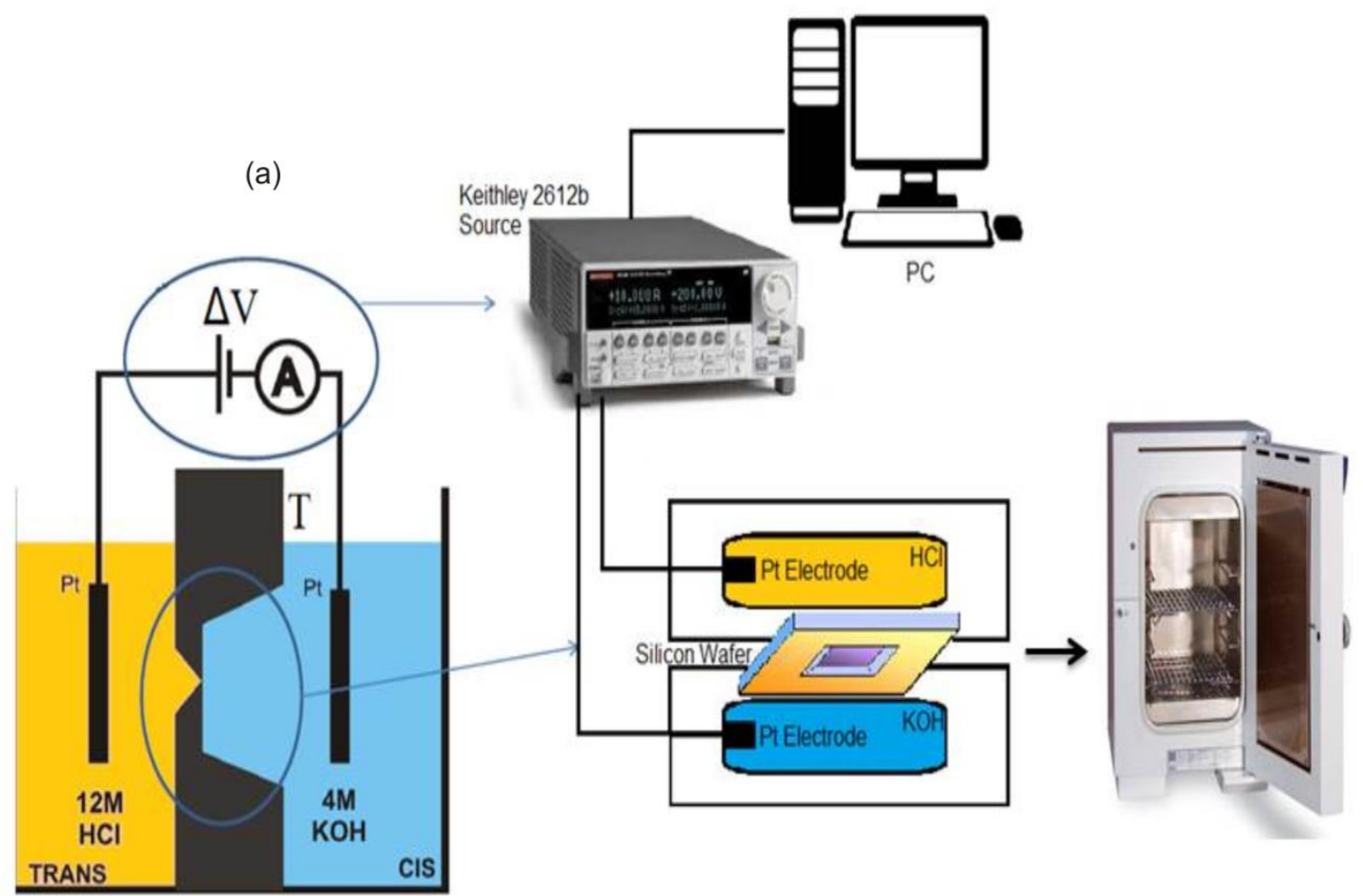

Figura 2: Ilustración esquemática: segunda etapa de etching químico. a) Celda con dos compartimientos para solución de etching $\mathrm{KOH} 4 \mathrm{M}$ y solución de frenado (HCL 12 M ó HCOOH 30M), b) Conexión de electrodos de platino conectados con un dispositivo Keithley 2612 b.

\section{RESULTADOS}

Se estudió la influencia de factores como la temperatura, el voltaje y el agente de frenado durante el wet etching, a fin de caracterizar el proceso de formación de poros. Al respecto, SEIDEL et al. [17], y CHEN et al [8], reportaron que el aumento de la temperatura incrementa la velocidad del ataque químico. Por otra parte, Harrel et al. [18], mostraron que el incremento del voltaje aplicado al proceso electroquimico genera un aumento del tamaño de poro. Recientemente, APEL et al. [12], reportaron que las características químicas del agente de frenado afecta la geometría del poro. En cuanto a los perfiles de corriente y tiempo, PARK et al. [19], reportaron que antes de la formación del poro, se genera fluido de corriente ("background") que es posible minimizar aplicando un voltaje de $800 \mathrm{mV}$. En base a lo anterior, se han variado las temperaturas del proceso $\left(65^{\circ} \mathrm{C}\right.$ y $\left.84^{\circ} \mathrm{C}\right)$, y se han establecido voltajes de $0,1 \mathrm{~V}, 0,5 \mathrm{~V}$ y $1 \mathrm{~V}$ respectivamente. En relación al agente de frenado, se han realizado experimentos con $\mathrm{HCl} 12 \mathrm{M}$ y $\mathrm{HCOOH} 30 \mathrm{M}$.

\subsection{Caracterización del proceso de fabricación de poros: $\mathrm{HCl}, \mathrm{KOH} 4 \mathrm{M}, 84{ }^{\circ} \mathrm{C}$}

La figura 3 muestra los resultados de la evolución de las corrientes registradas durante el wet etching obtenido bajo las siguientes condiciones: $84{ }^{\circ} \mathrm{C}, 1 \mathrm{~V}$ y HCl $12 \mathrm{M}$ como agente de frenado (C-84-1, según la tabla 1). Como es evidente, en la gráfica se pueden reconocer, posterior a la estabilización inicial, tres etapas compatibles con los fenómenos de movimiento iónico predominantes durante el ataque químico; la apertura y la neutralización.

En una primera instancia, previa a las $2 \mathrm{~h}$ de medición, se observa un ascenso sostenido y relativamente ordenado de la corriente eléctrica, compatible con la etapa de ataque químico esperada antes de apertura del poro. Durante este lapso, el comportamiento de la corriente se relaciona con la generación de electrones debidos a la reacción química entre el silicio y el KOH [15,18]. Alrededor de 1,76 horas después del inicio del proceso se observa un cambio en el comportamiento del gráfico: un nuevo ascenso, más desordenado y con menor rapidez. Al momento en que ocurre este primer cambio se le ha denominado $T_{1}$.

Se encontró que el momento en que finaliza esta primera etapa, $\mathrm{T}_{1}$, donde se encuentran las etapas 1 y 
2, es comparable el tiempo teórico correspondiente a la apertura del poro, según la ecuación 1 (tabla 2).

$$
T_{1} \equiv T_{R}=\frac{\left[D_{0}-D_{1}\right]}{V}
$$

Donde $\mathrm{D}_{0}=520 \mu \mathrm{m}$ es el espesor de oblea, $\mathrm{D}_{1}$ es la profundidad alcanzada durante el primer etching y $\mathrm{V}=1,40 \mu \mathrm{m} / \mathrm{min}$, es la velocidad de etching químico a $84{ }^{\circ} \mathrm{C}$ con $\mathrm{KOH} 4 \mathrm{M}$ [17]. Es por esta correspondencia que la etapa 2 , relativa a $\mathrm{t}>\mathrm{T}_{1}$, se ha denominado apertura del poro.

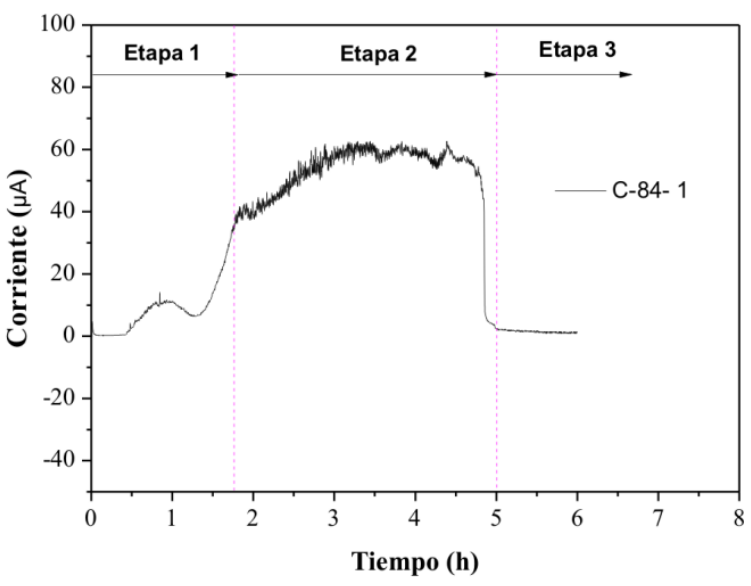

Figura 3: Evolución de la corriente para la condición C-84-1I. $T_{1}, T_{2}$ y $T_{3}$ corresponden con los tiempos característicos del proceso.

La figura 4 muestra de forma más detallada las etapas del proceso. Previo a las 2 h de medición, se observa un ascenso sostenido y relativamente ordenado de la corriente eléctrica que corresponde con el ataque químico (Figura 4a). Durante el inicio de apertura del poro la corriente sufre un incremento exponencial debido al flujo adicional de iones por el poro, como se puede observar figuras $4 \mathrm{~b}$ y $4 \mathrm{c}$ (este último gráfico corresponde a una linealización de la dependencia). Para finalizar, una vez que la neutralización y el frenado del wet etching son suficientemente importantes ( $\mathrm{t}>\mathrm{T}_{2}$ ), la corriente presenta un marcado decaimiento, dando por finalizado el proceso y deteniendo la apertura del macroporo, como se puede observar en la figura $4 \mathrm{~d}$, que resalta la etapa final del mismo. Es importante indicar que se ha denominado como etapa de neutralización y frenado del wet etching al momento que, para el experimento C-84-1, comienza a las 4,74 horas del proceso y para el cual el ácido clorhídrico $12 \mathrm{M}$ y el KOH $4 \mathrm{M}$ han estado en contacto durante aproximadamente 3 horas. Debido a este fenómeno, dichos compuestos reaccionan dando lugar a sales de KCl. Adicionalmente, la concentración de $\mathrm{KOH}$ disminuye y por tanto el ataque a la superficie del silicio es mucho menor en comparación con las condiciones iniciales del experimento. 


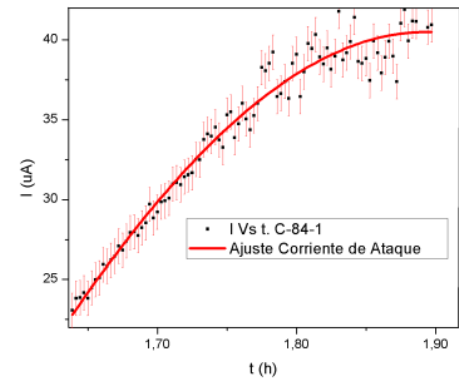

c)

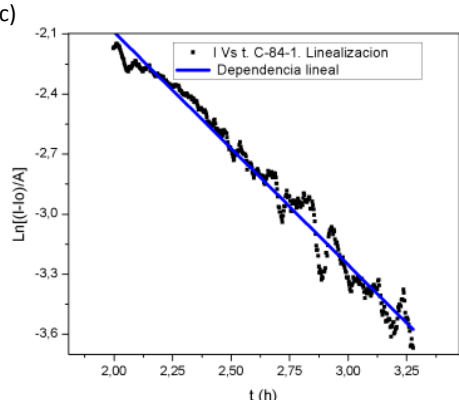

b)

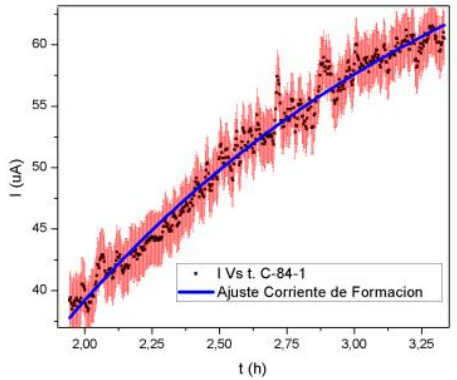

d)

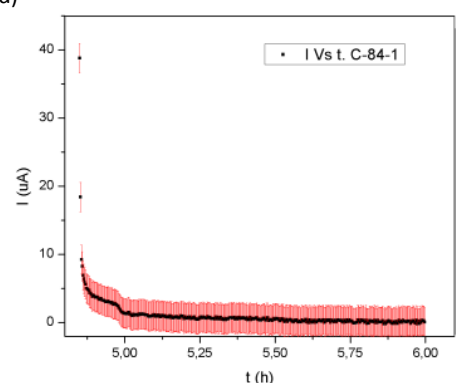

Figura 4: Descripción de las etapas del proceso de fabricación de poros. a) Comportamiento inicial correspondiente al ataque químico, b) Dependencia exponencial luego de apertura de poro, c) Trasformación exponencial del grafico 4b, d) Etapa de neutralización.

A partir de las anteriores consideraciones, se realizó una comparación del comportamiento de los experimentos realizados a $84{ }^{\circ} \mathrm{C}$ bajo diferentes voltajes, y con $\mathrm{HCl}$ como agente de frenado (Figura 5). En los gráficos de evolución de corrientes para las tres condiciones $(0,1 \mathrm{~V}, 0,5 \mathrm{~V}$ y $1 \mathrm{~V})$ se observan comportamientos similares, en donde se pueden diferenciar las 3 etapas discutidas previamente, y además, las corrientes de las 3 curvas resultan razonablemente proporcionales al voltaje utilizado para cada experiencia. En particular, las estabilizaciones intermedias de cada experimento en torno a $55 \mu \mathrm{A}, 25 \mu \mathrm{A}$ y $5 \mu \mathrm{A}$ respectivamente confirman esta suposición. Además, al igual que en la condición C-84-1, se encontró que para las condiciones C84-0.5 y C-84-0.1, el instante $\mathrm{T}_{1}$ es comparable con el tiempo teórico de apertura de poro calculado según la ecuación 1 (tabla 2). Por otro lado, en el grafico obtenido para la oblea C-84-0.5, se observa que a las 3,5 horas ocurre una disminución drástica de la corriente. Este comportamiento coincide con la apertura de un segundo poro en la misma oblea, lo que incrementa la velocidad de neutralización.

(a)

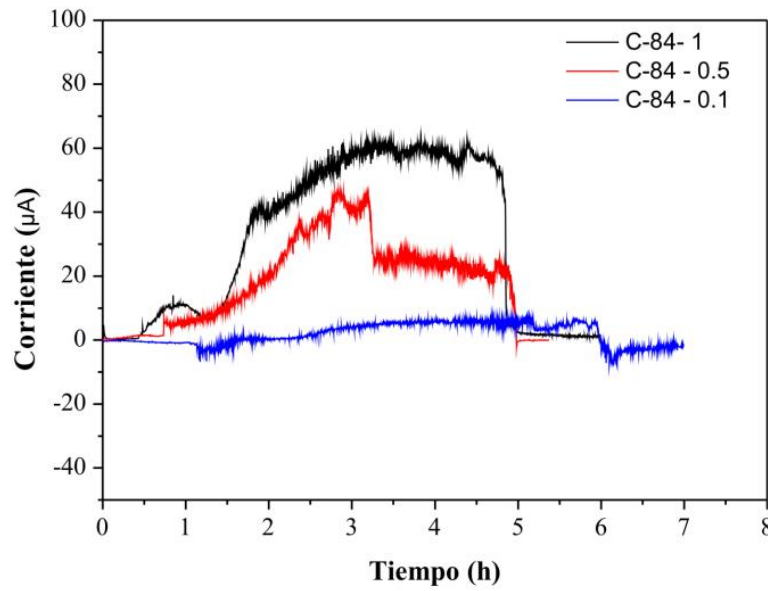

(b)

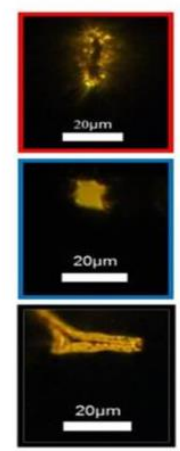

Figura 5: Comparación de las curvas de evolución de la corriente, condiciones: $\mathrm{HCl}, 84^{\circ} \mathrm{C}$. a) Línea negra: condición 1 V, Línea roja: condición $0,5 \mathrm{~V}$ y Línea azul: condición $0,1 \mathrm{~V}$, b) Imágenes de los macroporos obtenidos. 
APEL etal. [19] indicaron que aplicando voltajes mayores a 0,1 V, la electromigración es por lo menos 4 veces mayor que la difusión (este número asciende a 35 si se utiliza $1 \mathrm{~V}$ de potencial). El aumento del voltaje genera por tanto un aumento en la importancia de electromigración de iones $\mathrm{OH}$ - frente a mecanismos difusivos. Por esta razón, tanto los valores de corrientes obtenidos como las dependencias específicas de cada etapa son más visibles con el aumento del voltaje aplicado. A voltajes altos, entonces, este fenómeno permite distinguir con claridad las regiones mencionadas, y realizar análisis e hipótesis a partir de supuestos puramente eléctricos.

Tabla 2: Tiempo de apertura de poro teórico y experimental para tres obleas tratadas a distintas condiciones.

\begin{tabular}{l|l|l|l}
\hline OBLEA & $\begin{array}{l}\text { PROFUNDIDAD TOTAL OBTENIDO } \\
\text { DEL PRIMER ETCHING }\end{array}$ & $\begin{array}{l}\text { TIEMPO DE } \\
\text { APERTURA }^{1}\end{array}$ & $\begin{array}{l}\text { TIEMPO DE } \\
\text { APERTURA }^{2}\end{array}$ \\
\hline C-84-1 & 365 & 1,84 horas & 1,8 horas \\
\hline C-84-0.5 & 315 & 2,44 horas & 2,4 horas \\
\hline C-84-0.1 & 310 & 2,5 horas & 2,8 horas \\
\hline
\end{tabular}

1. Valor determinado según ecuación 1.

2. Dato obtenido de la curva de evolución de corrientes, figura 5.

Para determinar la reproducibilidad de los experimentos se han llevado a cabo 2 fabricaciones bajo las condiciones de $\mathrm{HCl}, 84{ }^{\circ} \mathrm{C}, 0,5 \mathrm{~V}$. La figura 6 muestra los gráficos de corriente eléctrica obtenidos durante proceso. Se encontró que, bajo estas condiciones, el instante $\mathrm{T}_{1}$ (tiempo de apertura de poro) coincidió con el tiempo de apertura obtenido a partir de la ecuación 1. Para la oblea C-84-0.5 replica 1 correspondió con 2,9 horas y para C-84- 0.5 replica 2 correspondió con 2,7 horas.

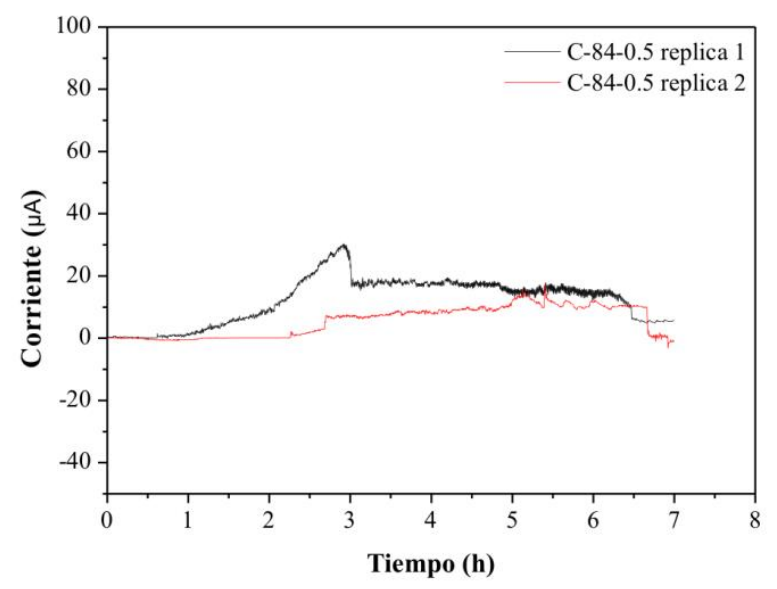

Figura 6: Comparación de las curvas de evolución de la corriente, condiciones: $\mathrm{HCl}, 84{ }^{\circ} \mathrm{C}$. Línea negra: 0,5 V-replica 1, Línea roja: 0,5 V-replica 2.

Tabla 3: Tiempo de apertura de poro teórico y experimental para tres obleas tratadas a distintas condiciones.

\begin{tabular}{l|l|l|l}
\hline OBLEA & $\begin{array}{l}\text { PROFUNDIDAD TOTAL OBTENIDA } \\
\text { DEL PRIMER ETCHING }\end{array}$ & $\begin{array}{l}\text { TIEMPO DE } \\
\text { APERTURA }^{1}\end{array}$ & $\begin{array}{l}\text { TIEMPO DE } \\
\text { APERTURA }^{2}\end{array}$ \\
\hline C-84-0.5 replica 1 & 280 & 2,85 horas & 2,9 horas \\
\hline C-84-0.5 replica 2 & 280 & 2,85 horas & 2,7 horas \\
\hline
\end{tabular}

1. Valor determinado según ecuación 1.

2. Dato obtenido de la curva de evolución de corrientes, figura 6. 


\subsection{Caracterización del proceso de fabricación de poros: $\mathrm{HCOOH}, \mathrm{KOH} 4 \mathrm{M}, 84^{\circ} \mathrm{C}$}

En la figura 7 se muestran los resultados obtenidos para los experimentos F-84-1, F-84-0.5 y F-84-0.1. En este caso, el proceso de fabricación de poros se llevó a cabo siguiendo el procedimiento utilizado en el apartado 3.1, con ácido fórmico como agente de frenado. En todos los casos de la figura se observan valores correspondientes a tres etapas bien definidas. En primer lugar, una evolución ordenada de la corriente, relacionada con la reacción química entre el silicio y el $\mathrm{KOH}[17,20]$. Luego se registra una fuerte fluctuación, cuya magnitud media resulta directamente proporcional al voltaje aplicado. Y finalmente una estabilización final, que concluye con el proceso.

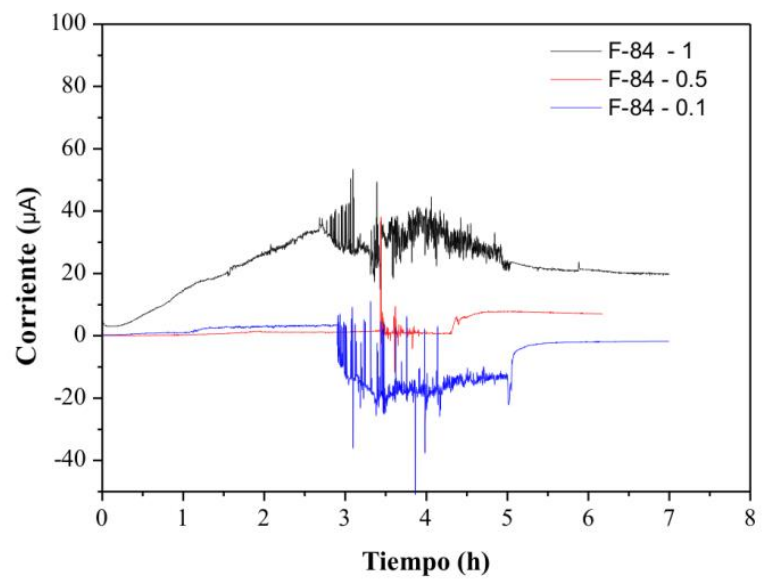

Figura 7: Comparación de las curvas de evolución de la corriente, condiciones: $\mathrm{HCOOH}, 84^{\circ} \mathrm{C}$. a) Línea negra: condición $1 \mathrm{~V}$, Línea roja: condición $0,5 \mathrm{~V}$ y Línea azul: condición $0,1 \mathrm{~V}$.

La tabla 4 muestra los valores de profundidad total obtenidos después del primer etching para las obleas F-84-1, F-84-0.5 y F-84-0.1. De acuerdo a estos valores, los tiempos de apertura según la ecuación 1 deberían ser inferiores a 2 horas. No obstante, para las obleas F-84-1, F-84-0.5 y F-84-0.1 se observaron cambios de magnitud de la corriente cerca de las 2,8 horas, 3,4 y 2,9 horas respectivamente. Para el caso específico de las obleas F-84-0.5 y F-84-0.1, además, el proceso no se frenó y la superficie de silicio fue atacada completamente. Dado que el experimento, por el contrario, se llevó a cabo durante aproximadamente 5,5 horas, un lapso suficiente para el desarrollo de las etapas de ataque, se puede atribuir a las propiedades químicas del ácido fórmico la ineficiencia del proceso de frenado.

Tabla 4: Tiempo de apertura de poro teórico y experimental para tres obleas tratadas a distintas condiciones.

\begin{tabular}{l|l|l|l}
\hline OBLEA & $\begin{array}{l}\text { PROFUNDIDAD TOTAL } \\
\text { OBTENIDA DEL PRIMER } \\
\text { ETCHING }\end{array}$ & $\begin{array}{l}\text { TIEMPO DE } \\
\text { APERTURA }^{1}\end{array}$ & $\begin{array}{l}\text { TIEMPO DE } \\
\text { APERTURA }^{2}\end{array}$ \\
\hline F-84-1 & 420 & 1,19 horas & 2,8 horas \\
\hline F-84-0.5 & 420 & 1,19 horas & 3,4 horas \\
\hline F-84-0.1 & 355 & 1,96 horas & 2,9 horas \\
\hline
\end{tabular}

1. Valor determinado según ecuación 1.

2. Dato obtenido de la curva de evolución de corrientes, figura 7.

La figura 8 muestra imágenes de macroporos tomadas mediante microscopía electrónica de barrido, obtenidas bajo las mismas condiciones experimentales anteriormente descriptas: $\mathrm{HCOOH}, 84{ }^{\circ} \mathrm{C} 1 \mathrm{~V}$. Se puede observar que cuando la ablación del nitruro de silicio coincide con la parte lateral de la ventana, la forma del poro obtenido es irregular, mientras que cuando la ablación del nitruro del silicio coincide con la parte interna de la ventana, éste toma forma cuadrada. 


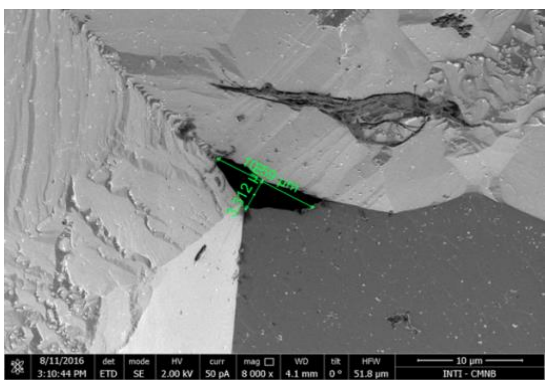

c)

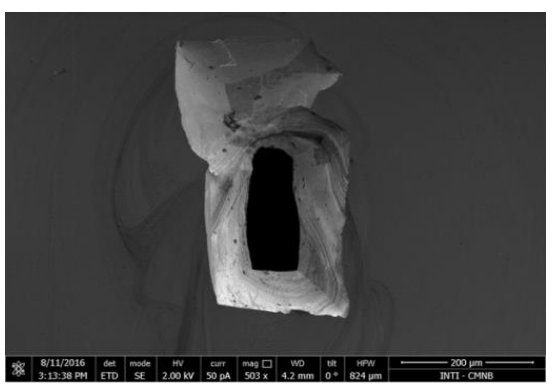

b)

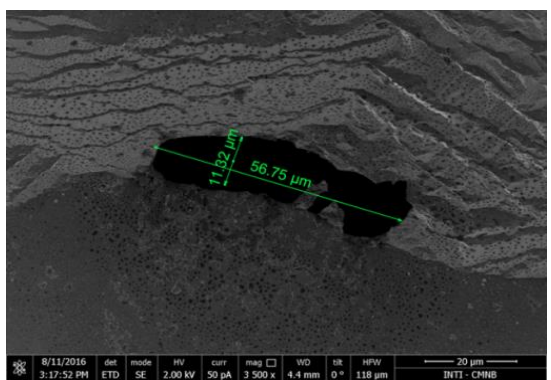

d)

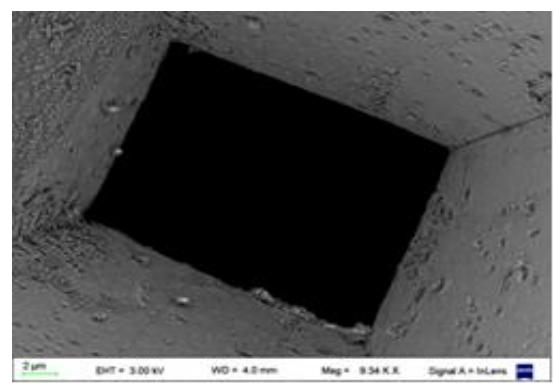

Figura 8: Imágenes de poros obtenidas mediante microscopia electrónica de barrido. a y b) macroporos formados en la parte lateral de la ventana, c y d) macroporos formados dentro de la ventana. Condiciones a: $\mathrm{HCOOH}, 84{ }^{\circ} \mathrm{C}, 1 \mathrm{~V}$.

\subsection{Caracterización del proceso de fabricación de poros: $\mathrm{HCOOH}, \mathrm{HCl}, \mathrm{KOH} 4 \mathrm{M}, 65{ }^{\circ} \mathrm{C}$}

SEIDEL et al.[17] indicaron que la velocidad de ataque químico es influenciada por la temperatura aplicada; Una menor temperatura genera menor velocidad, y a su vez la disminución de la velocidad permite un mayor control sobre el tamaño del poro obtenido. Por este motivo, la caracterización del proceso de fabricación de poros también se llevó a cabo a $65^{\circ} \mathrm{C}$.

La figura 9 exhibe las superficies de las obleas C-65-1, F-65-1, C-65-0.5, F-65-0.5, C-65-0.1 y F-650.1 obtenidas después del etching electroquímico. Aunque las obleas se expusieron entre 13 y 16 horas al ataque químico con $\mathrm{KOH}$, aplicando voltajes de $1 \mathrm{~V}, 0,5 \mathrm{~V}$ y $0,1 \mathrm{~V}$ respectivamente, no se obtuvieron poros en ninguno de los casos. De acuerdo con la velocidad de ataque del $\mathrm{KOH}$ a $65{ }^{\circ} \mathrm{C}$, se debería alcanzar una profundidad entre 400 y $500 \mu \mathrm{m}$, lo cual corresponde a una profundidad en exceso para todas las obleas. En la tabla 5 se indican las profundidades finales obtenidas posteriores al ataque químico, se puede observar que la profundidad de ataque se encuentra entre $20 \mu \mathrm{m}$ y $100 \mu \mathrm{m}$. Estos resultados indican que posiblemente a la temperatura de $65{ }^{\circ} \mathrm{C}$ se genera un alto grado de burbujas de hidrógeno producidas en la reacción $(\mathrm{Si}+$ $\left.2(\mathrm{OH})^{-}+4 \mathrm{H}_{2} \mathrm{O} \rightarrow \mathrm{Si}(\mathrm{OH})_{6}^{-}+2 \mathrm{H}_{2}\right)$, que enmascaran la superficie y que da lugar a la disminución de la velocidad de ataque químico $[17,20]$.

Por otro lado, en las imágenes también se observa que la mayoría de las superficies presentan rugosidad, lo que es congruente con los resultados obtenidos por PALIK et al. [20], quienes indicaron que la disminución de la temperatura de ataque químico se genera un mayor grado de rugosidad por un aumento de formación de silicatos. 


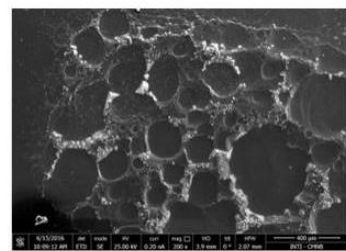

C-65-1

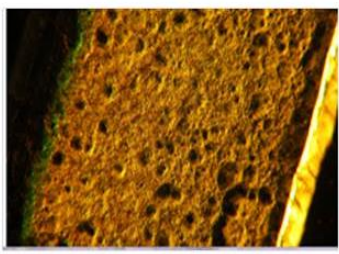

F-65-1

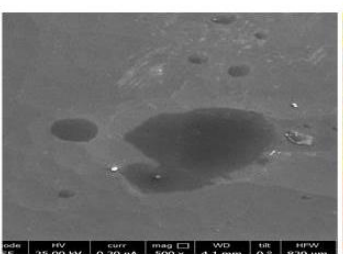

C-65-0.5

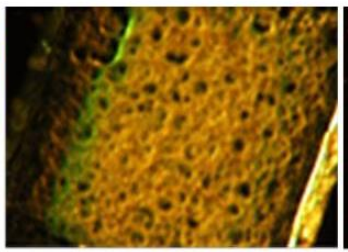

F-65-0.5

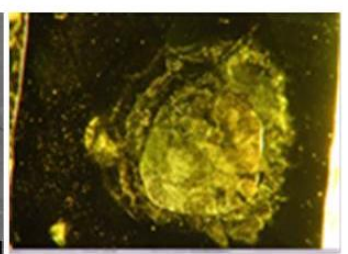

C-65-0.1

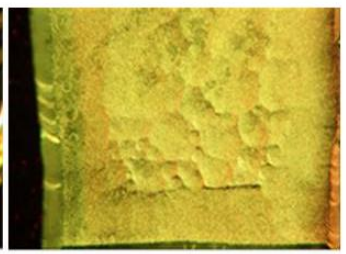

F-65-0.1

Figura 9: Superficie obtenida posterior al proceso electroquímico de formación de poros. Condiciones: $\mathrm{KOH} 4 \mathrm{M}$, $\mathrm{HCOOH} 30 \mathrm{M}$, Temperatura: $65^{\circ} \mathrm{C}$.

Tabla 5: Tiempo de apertura de poro

\begin{tabular}{l|l|l|l}
\hline OBLEA & $\begin{array}{l}\text { PROFUNDIDAD TOTAL } \\
\text { OBTENIDA DEL } \\
\text { PRIMER ETCHING }\end{array}$ & $\begin{array}{l}\text { PROFUNDIDAD TOTAL } \\
\text { OBTENIDA DEL SEGUNDO } \\
\text { ETCHING }\end{array}$ & $\begin{array}{l}\text { TIEMPO } \\
\text { APROXIMADO DE } \\
\text { APERTURA }^{1}\end{array}$ \\
\hline F-65-1 & 400 & 500 & 4 horas \\
\hline F-65-0.5 & 400 & 490 & 4 horas \\
\hline F-65-0.1 & 360 & 400 & 5.3 horas \\
\hline C-65-1 & 480 & 500 & 1.3 horas \\
\hline C-65-0.5 & 440 & 500 & 2.7 horas \\
\hline C-65-0.1 & 420 & 500 & 3.3 horas \\
\hline
\end{tabular}

1. Valor determinado según ecuación 1.

\section{DISCUSIÓN}

De acuerdo con los resultados de la literatura, la velocidad de ataque está relacionada con la variación de las condiciones de fabricación, tales como el voltaje [18], la temperatura [17,20] y la concentración de agente de ataque químico [17,20]. SEIDEL et al. [17] reportaron el efecto de la temperatura y la concentración de KOH en la velocidad del ataque químico. La velocidad de ataque aumenta antes de una concentración de KOH 20$30 \%$, luego disminuye; También reportó que el aumento de la temperatura produce un aumento en la velocidad de ataque químico. Adicionalmente, HARREL et al. [18] mostraron una relación directa entre el voltaje aplicado y el tamaño del poro. En relación con el proceso de caracterización, se ha reportado que no existe corriente de fondo cuando el ataque químico se hace sobre materiales como politereftalato de etileno [21], polimida [23], policarbonato [24].

En contraste, cuando el material es silicio, se genera corriente de fondo por efecto de la generación de electrones que se producen en la reacción entre el $\mathrm{KOH}$ y el silicio $(\mathrm{Si}+4 \mathrm{OH} \rightarrow \mathrm{Si}(\mathrm{OH}) 4+4 \mathrm{e}-)$. $\mathrm{Al}$ respecto, PARK etal. [19] reportaron que en sus ensayos se minimizó este fondo de corriente aplicando voltajes de $800 \mathrm{mV}$, por efecto la diferencia de potencial electroquímico entre las interfaces $\mathrm{KCl} / \mathrm{Si}$ y $\mathrm{KOH} / \mathrm{Si}$ [17]. En general, los estudios anteriores indicaron que el voltaje, la temperatura y la concentración de KOH son factores clave en el tamaño del poro y en la caracterización del proceso de fabricación. En cuanto a los resultados obtenidos en el presente trabajo, se pudo observar que bajo las condiciones estudiadas los perfiles de corriente en función del tiempo presentan valores de fondo que se minimizan cuando se aplica voltajes de $0,1 \mathrm{~V}$. Por otra parte, las dependencias relativas a la caracterización de la fabricación de macroporos bajo las condiciones de $\mathrm{HCl}, 84^{\circ} \mathrm{C}$ y voltajes de $1 \mathrm{~V}, 0,5 \mathrm{~V}$ y $0,1 \mathrm{~V}$, permiten diferenciar 3 tiempos característicos en el proceso, correspondientes a las etapas de ataque químico, apertura y neutralización, que pueden ser determinados a 
partir de las curvas de evolución de corrientes. En este sentido, la comparación entre el tiempo de apertura teórico y el tiempo de apertura experimental pone de manifiesto que es posible caracterizar el proceso de fabricación de poros sobre este sustrato. Por otra parte, el conocimiento de cada una de las etapas permitió determinar el momento de apertura del poro, lo que resulta de gran utilidad para la fabricación de nanoporos, ya que el proceso se detendría al instante denominado como $\mathrm{T}_{1}$.

Por otro lado, mediante la metodología utilizada es posible obtener poros de diferentes tamaños, ya que el proceso modula dicho parámetro en función del tiempo en que el $\mathrm{KOH} 4 \mathrm{M}$ está en contacto con la superficie del silicio. En lo relacionado con los experimentos a $84{ }^{\circ} \mathrm{C}$ con $\mathrm{HCOOH}$, los perfiles de corriente tiempo no permitieron determinar el momento de formación del poro. Además, cabe destacar que características particulares de este ácido, como su dificultad para disociarse, vuelven menos efectivo el mecanismo de frenado, dando lugar a 1) ataques químicos en toda la superficie de silicio de la oblea, lo que genera una ventana de características macroscópicas, y por tanto 2) No es posible tener un control del tamaño del poro. Respecto a los resultados obtenidos bajo las condiciones de $65^{\circ} \mathrm{C}$, con $\mathrm{HCOOH}$ y $\mathrm{HCl}$ como agentes de frenado, se puede indicar que en estos casos no fue posible un eficiente ataque químico al silicio cristalino y por tanto obtener poros ni caracterizar el proceso bajo estas condiciones.

\section{CONCLUSIONES}

El estudio de los perfiles de evolución de las corrientes ha puesto en evidencia que es posible la fabricación de poros de forma controlada. Se encontró que las condiciones de voltaje, reactivos y temperaturas aplicadas durante el proceso de formación influyen sobre características relevantes de la fabricación, determinándose las condiciones óptimas para una temperatura de $84{ }^{\circ} \mathrm{C}$ y $\mathrm{HCl}$ como agente de frenado. Por el contrario, bajo condiciones de temperatura a $65^{\circ} \mathrm{C}$ no se genera un ataque eficiente de la superficie del silicio y por tanto no es posible obtener poros en esta situación. Finalmente, el análisis de la evolución de las corrientes del proceso para condiciones óptimas de la fabricación permite caracterizar la formación macroporos y ofrece un mecanismo altamente funcional para el control de su tamaño.

En general el trabajo ofrece las siguientes posibilidades:

1. Caracterizar el proceso de fabricación de macroporos bajo las condiciones establecidas

2. Determinar condiciones óptimas de fabricación de macroporos

3. Provee una metodología económica, fácil y versátil para la fabricación de macroporos

4. Lo anterior da lugar a la extrapolación y adecuación del procedimiento en una metodología que permite la fabricación de nanoporos, generando así una herramienta de utilidad para el desarrollo tecnológico, tanto de la medicina como de la biología.

\section{AGRADECIMIENTOS}

Este trabajo ha sido financiado por CONICET-YPF (PIO-2014), ANPCyT (PICT2012-575/PICT2013- 0840) y FAN 2014, Argentina.

\section{BIBLIOGRAFÍA}

[1] HAQUE, F., LI, J., WU, H.-C., et al., "Solid-state and biological nanopore for real-time sensing of single chemical and sequencing of DNA", Nano Today, v. 8, pp. 56-74, Feb.2013.

[2] LIU, S., ZHAO, Q., LI, Q., ZHANG., H, et al., "Controlled deformation of Si $3 \mathrm{~N} 4$ nanopores using focused electron beam in a transmission electron microscope", Nanotechnology, v. 22, pp. 113201-1/113201-4,Sept 2011.

[3] LANYON, Y. H., MARZI, D.G., WATSON, Y.E., et al.,'Fabrication of Nanopore Array Electrodes by Focused Ion Beam Milling”, Analytical Chemistry, v.79, pp. 3048-3055, March 2007.

[4] SPINNEY, P.S., HOWITT, D.G., SMITH, R.L., et al., "Nanopore formation by low-energy focused electron beam machining”, Nanotechnology, v. 21, n.37, PP. 375301, Aug. 2010.

[5] GADGIL, V,J.,TONG, H,D.,CESA, Y., et al., "Fabrication of nano structures in thin membranes with focused ion beam technology”, Surface and Coatings Technology, v. 203, pp.2436-2441, June, 2009.

[6] STORM, A. J., CHEN, J. H., LING, X. S., et al., "Fabrication of solid-state nanopores with singlenanometre precision", Nat Mater, v. 2, p. 537-540, July 2003.

[7] VEGA, M., LERNER, B., LASORSA, C. A., et al., "Automated and low cost method to manufacture 
addressable solid-state nanopores", Microsystem Technologies, v. 22, pp. 109-117, Dic 2014.

[8] CHEN, J., DENG, T., WU, C. N., et al., "Fabrication of Silicon Nanopore Arrays with Three-Step Wet Etching", ECS Transactions, v. 52, pp.371-376, Aug. 2013.

[9] YANAGI, I., AKAHORI, R., HATANO, T., et al., "Fabricating nanopores with diameters of sub-1 nm to $3 \mathrm{~nm}$ using multilevel pulse-voltage injection”, Scientific Reports, v. 4, pp 1-7, May. 2014.

[10] BRIGGS, K., KWOK, H., TABARD-COSSA, V. "Automated Fabrication of 2-nm Solid-State Nanopores for Nucleic Acid Analysis", Small, v.10, pp. 2077 - 2086, March. 2014.

[11] KUAN, A.T., LU, B., SZALAY,T., et al, "Electrical pulse fabrication of graphene nanopores in electrolyte solution", Applied Physics Letters, v. 106, 203109 -1 -203109 -9, May. 2015.

[12] APEL, P. Y., BASHEVOY, V., BLONSKAYA, I., et al.,"Shedding light on the mechanism of asymmetric track etching: an interplay between latent track structure: etchant diffusion and osmotic flow", Phys. Chem. Chem. Phys, v. 18, pp. 25421-25433 Aug. 2016.

[13] SEXTON, L., MARTIN L.P. "Developing synthetic conical nanopores for biosensing applications", Mol. BioSyst., v. 3, pp. 667-685, September 2007.

[14] HOLZE, R. "Electrochemistry of silicon and its oxides", Journal of Solid State Electrochemistry, v.7, pp. 318-319, April 2003.

[15] SATO, K.,SHIKIDA, M., MATSUCHIMA, Y., et al., "Characterization of orientation-dependent etching properties of single-crystal silicon: effects of $\mathrm{KOH}$ concentration", Sensors and Actuators A, v. 64, pp. 87-93, 1988.

[16] COLLINS, S., "Etch Stop Techniques for Micromachining", Journal of the Electrochemical Society, v. 144. n.6, pp. 2242-262, 1997.

[17] SEIDEL, H., CSPREGI, L.,HEUBERGER,A., et al., "Anisotropic Etching of Crystalline Silicon in Alkaline Solutions, I. Orientation Dependence and Behavior of Passivation Layers", J. Electrochem. Soc, v.137, pp. 3612-3626, Nov 1990.

[18] HARRELL, C. C., SIWY, Z. S., MARTIN, C. R. "Conical Nanopore Membranes: Controlling the Nanopore Shape", Small , v. 2, pp. 194-198, Nov. 2006.

[19] PARK, S. R., PENG, H., LING, X. S. "Fabrication of Nanopores in Silicon Chips Using Feedback Chemical Etching", Small , v.3, pp. 116-119, Oct. 2006.

[20] CHEN, L., CHEN, M., LIEN, C., et al., "The Band Model and the Etching Mechanism of Silicon in Aqueous KOH”, Journal of The Electrochemical Society, v. 142, pp. 170-176. Jun.1995.

[21] APEL, P. Y., KORCHEV, Y. E., SIWY, Z., et al., "Diode-like single-ion track membrane prepared by electro-stopping. Nuclear Instruments and Methods in Physics Research", Section B: Beam Interactions with Materials and Atoms, v. 184, pp. 337-346, Nov2001.

[22] PALIK, E. D., GLEMBPCKI, O. J., HEARD, I., et al., "Etching roughness for (100) silicon surfaces in aqueous KOH", Journal of Applied Physics, v.70, Oct 1991.

[23] SIWY, Z., DOBREV, D., NEUMANN, R., et al., "Electro-responsive asymmetric nanopores in polyimide with stable ion-current signal", Applied Physics A , v.76, pp.781-785, March 2003.

[24] CHTANKO, N., TOIMIL MOLARES, M. E., CORNELIUS, T., et al., "Etched Single-Ion-Track Templates for Single Nanowire Synthesis", The Journal of Physical Chemistry B, v. 108, pp. 9950-9954, June 2004. 